\title{
Marketing digital para la fidelización de los clientes de la línea sindical de transporte
}

\author{
Digital marketing for the loyalty of customers of the union transport line
}

Marketing digital para fidelização de clientes da linha sindical de transporte

Recibido: enero 2021

Arbitrado: febrero 2021

Aceptado: marzo 2021

Publicado: mayo 2021
Juvenal Otondo Sardinas

juvenaloto@hotmail.com

https://orcid.org/0000-0001-8776-0624

Universidad Privada “Domingo Savio”, Potosí-Bolivia

\section{RESUMEN}

La investigación tuvo como objetivo contribuir con la fidelización de clientes de la línea Sindical Trans. Emperador de la ciudad de Potosí. La metodología fue de tipo descriptivo, con un enfoque mixto, y un diseño documental de campo. Los métodos utilizados fueron análisissíntesis, inductivo- deductivo, y comparativo; como técnicas e instrumentos se empleó, la entrevista y encuesta. La población fue 115025 personas mayores de 18 años y la muestra, fue de 383 personas. Como resultado se obtuvo que, los socios de la empresa indicaron que no se hace un control, ya que, no cuentan con un registro de clientes. Por lo que se concluye, que con la propuesta harán uso de las herramientas como; elaboración de una página web, estar presente en Facebook, entre otros. De esta manera, se podrá tener un mejor control respecto a la fidelización de los clientes de la línea Sindical de la ciudad de Potosí.

\footnotetext{
Palabras clave: Marketing; marketing digital; fidelización,
}

The objective of the investigation was to contribute to the loyalty of clients of the Sindical Trans line. Emperor of the city of Potosí. The methodology was descriptive, with a mixed approach, and a field documentary design. The methods used were analysissynthesis, inductive-deductive, and comparative; As techniques and instruments, the interview and survey were used. The population was 115,025 people over 18 years of age and the sample was 383 people. As a result, it was obtained that the partners of the company indicated that a control is not carried out, since they do not have a customer registry. Therefore, it is concluded that with the proposal they will use tools such as; development of a web page, be present on Facebook, among others. In this way, it will be possible to have a better control regarding the loyalty of the clients of the Union line of the city of Potosí.

Palabras clave: Marketing; digital marketing; loyalty, customer

\section{RESUMO}

O objetivo da investigação foi contribuir para a fidelização dos clientes da linha Sindical Trans. Imperador da cidade de Potosí. A metodologia foi descritiva, com abordagem mista, e desenho documental de campo. Os métodos utilizados foram análise-síntese, indutivo-dedutivo e comparativo; Como técnicas e instrumentos, utilizou-se a entrevista e o inquérito. A população era de 115.025 pessoas com mais de 18 anos e a amostra era de 383 pessoas. Como resultado, obteve-se que os sócios da empresa indicaram que não é realizado o controle, por não possuírem cadastro de clientes. Portanto, conclui-se que com a proposta utilizarão ferramentas como; desenvolvimento de página web, presença no Facebook, entre outros. Desta forma, será possível ter um melhor controle quanto à fidelização dos clientes da linha Union da cidade de Potosí.

Palabras clave: Marketing; marketing digital; lealdade, cliente 


\section{INTRODUCCIÓN}

$\mathrm{E}$ marketing se ha convertido en un elemento fundamental para coordinar las actividades empresariales correctamente a través de la orientación al cliente; este principio permite desarrollar un direccionamiento estratégico organizacional que guie a la empresa no solamente a vender un producto o servicio, sino más bien a estar atenta a ofrecer soluciones a las necesidades y satisfacción de los deseos del mercado meta.

La importancia del Marketing, según Kotler y Armstrong (2012), consiste en un proceso administrativo y social, gracias a determinados grupos o individuos que obtienen lo que necesitan o desean a través del intercambio de productos o servicios. Por este hecho, las empresas dependerán en gran medida del manejo adecuado del marketing para poder consolidarse en el mercado y lograr que el cliente se fidelice con el producto o servicio.

Cabe recalcar que hoy en día los medios digitales son cada vez más tomados en cuenta para una planeación integrada de mercadeo: redes sociales, marketing en buscadores, optimización web, email marketing, video y móvil marketing, los cuales son algunos de los formatos y herramientas de las que dispone un profesional de mercadeo digital. Se debe considerar que hoy en día las empresas no llegan a los clientes, sino ellos llegan a éstas mediante las nuevas tecnologías de comunicación que van en constante evolución.

Se debe considerar que ante el gran nivel de competitividad empresarial la fidelización de los clientes meta, es un objetivo fundamental que las organizaciones deben tomar en cuenta, ya que, mediante la misma se trata de asegurar la relación continuada del cliente con la empresa, evitando que sea alcanzado por la competencia. Es decir, es un objetivo fundamental del marketing el mantener y crear un vínculo con los clientes nuevos y antiguos de la empresa.

La importancia de la fidelización de clientes tiene como objetivo conseguir, mediante diversas estrategias y tácticas de marketing, que el cliente que haya adquirido con anterioridad algunos de los productos o servicios de la empresa siga comprando y se convierta un cliente habitual.

De esta manera, la investigación tiene como objetivo, desarrollar tácticas de marketing digital para la fidelización de los clientes de la línea Sindical Trans Emperador de la ciudad de Potosí.

En este sentido, es importante tener tácticas de marketing digital para poder fidelizar al cliente porque se daría a conocer los servicios, rutas que ofrece la línea Sindical Trans Emperador mediante las nuevas tendencias de la tecnología.

\section{Marketing}

Según Kotler y Armstrong (2012) el marketing se constituye como un proceso social y administrativo, que permite que los individuos, organizaciones, o grupos empresariales mejoren los indicadores en cuanto a sus necesidades creando e intercambiando valor con otros. En el contexto de negocios más estrecho, el marketing establece relaciones redituables, de intercambio de valor agregado, con los clientes. Es por ello, que se puede aproximar una definición sobre el marketing un como proceso a través del cual las compañías crean valor para sus clientes y establecen relaciones sólidas con ellos para obtener a cambio valor de éstos. 
De acuerdo a Lamb, Hair y Mc Daniel (2017), el marketing es la actividad, el conjunto de instituciones y los procesos para crear, comunicar entregar e intercambiar ofertas que tienen valor para los clientes, socios y la sociedad en general (p. 3).

\section{Marketing de servicios}

Los especialistas en marketing de servicios deben ser capaces de transformar los servicios intangibles en beneficios concretos y en una experiencia bien definida. En base a esto, Kotler y Armstrong (2012) plantea que los servicios son productos o bienes intangibles. En donde los temas acerca de los productos que se han analizado hasta ahora, se aplican tanto a los servicios como a los productos físicos. Teniendo como principal objetivo el enfoque en las características especiales y las necesidades de marketing que distinguen a los servicios. Aguirre (2006), también define al marketing de servicios como la aplicación del marketing en la concepción y comercialización de un producto intangible como núcleo del intercambio

Aunado a ello, las empresas que ofrecen servicios, hoy en día enfrentan una fuerte competencia. Muchos propietarios y gerentes se quejan de lo difícil que es mantener los costos bajos, obtener ganancias, encontrar empleados calificados y motivados a satisfacer a los clientes que, según ellos, se han vuelto exageradamente demandantes. Es así como, de una empresa a otra, la competencia estimula la innovación, mejoramiento especialmente a través de la aplicación de tecnologías nuevas y mejoradas. No sólo existe competencia entre empresas dentro del mismo rubro, sino también entre empresas que están en otros rubros y que pueden ofrecer nuevas soluciones a las necesidades de los clientes.

\section{Fidelización}

Según Alcaide (2002), es definida como, una actitud positiva, que supone la unión de la satisfacción del cliente (formada por elementos racionales, afectos y comportamientos) con una acción de consumo estable y duradera. (p. 7).

De este modo, la definición del autor anterior, es crear un vínculo que jinete la lealtad entre los clientes que ya obtuvieron un producto o servicio de la empresa, y que los mismos se conviertan en clientes frecuentes.

A su vez, los componentes de la fidelización pretenden establecer un vínculo a largo plazo entre la empresa y el cliente, y que la relación dure una vez finalizada la compra.

Según Agüero, (2014, pp. 17-18) algunos de los componentes de la fidelización son:

- Diferenciación, es la estrategia seguida por parte de la empresa que hace referencia a las características de nuestros artículos, presentándolos como únicos frente a los de la competencia. Distinción, valoración, equidad y proporcionalidad, son características fundamentales dentro de este tipo de estrategia.

- Personalización, cada cliente es diferente y requiere unas características de un determinado producto. Para configurarlo contamos con el propio cliente, que nos proporcionará las pautas de sus preferencias. Para realizar la personalización con éxito 
debemos de reconocer e identificar al cliente y con todo ello adaptar los artículos a sus necesidades.

- Satisfacción, todas aquellas características y dimensiones del producto que el cliente percibe y le producen un determinado placer.

- Fidelidad, compromiso por parte del cliente a la marca y por parte de la empresa hacia el cliente. Con esto se pretende que la empresa cumpla una serie de requisitos y promesas establecidas, es un paso importante hacia la fidelización del cliente.

- Habitualidad, frecuencia, volumen, cantidad, duración, con la que nuestros clientes realizan sus compras.

\section{Instrumentos y causas de la fidelización de clientes}

Para Alcaide (2002), existen varias causas que llevan a un cliente a mantenerse fiel a un producto o servicio. Entre las principales causas de fidelidad que las investigaciones se encuentran:

- Precio. Una primera causa de fidelidad es el precio. Pero en los servicios bancarios actuales las investigaciones demuestran que no es la razón fundamental para la selección de entidad bancaria

- Calidad. En la mayor parte de los productos y servicios la decisión de compra no se guía estrictamente por el precio sino por la calidad incluso aunque el producto físicamente sea el mismo, el consumidor puede percibido como distinto.

- Valor percibido. Es ese Valor percibido subjetivamente por el consumidor el que emplea para seleccionar ofertas.

- Imagen. El consumidor no es estrictamente racional sino que muy a lo contrario se suele guiar por percepciones subjetivas, por sentimientos, emociones y por diferentes rasgos de personalidad que asigna a los productos o servicios.

- No hay alternativas. La fidelidad en muchos casos se produce porque el consumidor no conoce o dispone de alternativas. El incremento de la competencia, hace que los consumidores tengan muchas alternativas y esto dificulta el mantenerlos fieles. (pp. 23-24)

\section{Cliente}

Según Kotler y Armstrong (2003), "vivimos en una economía en la que se considera que el cliente es el rey. Este hecho es el resultado de un exceso de capacidad productiva en las empresas" (p. 8).

Según Bastos (2006):

El cliente es la persona que adquiere un bien o servicio para uso propio o ajeno a cambio de un precio determinado por la empresa y aceptado socialmente. Constituye el elemento fundamental por y para el cual se crean productos en las empresas. (p. 2) 
Por cliente entendemos que es la persona, empresa u organización quien adquiere o compra de forma voluntaria productos o servicios que necesitan o desean para sí mismo, para otra persona o para una empresa u organización, por lo que, es el motivo principal por el que se crean, producen, fabrican y comercializan productos y servicios.

\section{Satisfacción del cliente}

Según Kotler y Armstrong (2003), "es el nivel de estado de ánimo de una persona que resulta de comparar el rendimiento percibido de un producto o servicio con sus expectativas" (p. 40).

Se puede decir que, la satisfacción sólo es un estado de ánimo que tiene un cliente cuando se llenan las expectativas. Por lo tanto, este elemento va estrechamente ligado a la percepción de un producto o servicio.

\section{Elementos que conforman la satisfacción del cliente}

Según Kotler y Armstrong (2003) la satisfacción del cliente está conformada por tres elementos:

- El rendimiento percibido. En este punto se refiere al desempeño que el cliente considera haber obtenido luego de adquirir un producto o servicio. Dicho de otra forma, es el resultado que el cliente percibe cuando obtuvo el producto o servicio.

- Las expectativas. Son las "esperanzas" que los clientes tienen por conseguir algo.

- Nivel de satisfacción. Luego de realizada la compra o adquisición de un producto o servicio, los clientes experimentan uno de estos tres niveles de satisfacción; insatisfacción, satisfacción y complacencia.

\section{Marketing digital}

El marketing digital es un tipo de marketing cuya función es mantener conectada a la empresa u organización con sus segmentos de mercado y clientes, mediante los medios digitales que estén disponibles, con la finalidad de comunicarse fluidamente con ellos, brindarles servicios y realizar actividades de venta. (Buchelli y Cabrera, 2017, p. 37).

Como bien se sabe hoy en día, el mundo se va globalizando cada vez más, y tiene una tendencia a la innovación y tecnología, donde los dispositivos electrónicos van ganando mayor importancia. El internet, después de mucho tiempo se ha convertido en un punto de referencia antes de poder realizar cualquier tipo de decisión.

De tal forma, no existe una barrera entre realizar marketing tradicional o marketing digital, sino una unión que debe ser considerada como una estrategia actualizada y dinámica que combina dos entornos en los cuales se conviven.

\section{Tipos de redes sociales}

Las redes sociales no son nada más ni menos que los lugares donde las personas pueden publicar, compartir todo tipo de información, ya sea de tipo personal como también profesional, con amigos, familiares, conocidos y desconocidos. 
Según Merodio (2000), indica que, las redes sociales se pueden categorizar en varias opciones en función de su utilidad y aplicación, y en este aspecto en misma multitud de variantes. Entre ellas cabe mencionar; redes sociales, publicaciones, RSS, móviles, entre otras.

- Redes sociales. Son herramientas que permiten compartir información con amigos, y esta información puede ser texto, audio, video. Entre ellos están; Facebook, Linkedln, My spane, Ning, otros.

- Publicaciones. Son plataformas que permiten compartir contenido con trabajadores, clientes o cualquier persona en la Red, de tal manera que se aporta un valor en el contenido que se publica. Entre ellos están, Blogger, Wikia, Wordpress, otros.

- RSS. Gracias al RSS se está al tanto de las últimas actualizaciones de contenido en la webs y blogs preferidos, al igual que puede usarse para que los clientes estén informados de todo lo que sucede en un negocio. Entre ellos están, FeedBurner by Google, Atom, PingShot y RSS 2.0

- Móviles. Los móviles se están convirtiendo en una extensión del ordenador la cual llevamos a todas horas con nosotros. Es por esto por lo que el Marketing Móvil está ganando cuota de mercado y cada día son más las empresas que aprovechan sus recursos. Entre ellos están, CallWave, airG, Jott, otros.

\section{Captación de clientes a través del marketing digital}

Según Escobar (2013), la captación de clientes mediante el marketing digital, se puede realizar de la siguiente manera;

- El cliente es lo más importante. Al cliente hay que darle la importancia que se merece, es decir, toda. Recuerda que tu empresa es lo que es gracias a él.

- Educación. Trata a tu cliente con la educación que te gusta que te traten y a ti y con la que se merecen. No olvides que eres el lazo de unión entre tu empresa y ellos y un buen trato e imagen es indispensable.

- Saber escuchar. Aquel que no sepa escuchar no sabrá nunca solucionarle una incidencia o solicitud a su cliente.

- Seguimiento. Mientras la solicitud o incidencia de tu cliente esté en trámites, infórmale. Ponte en contacto con él asiduamente para indicarle cómo va. Así tu cliente verá que te preocupas y que su problema no ha quedado en el olvido.

- Empatía. Punto básico y esencial para aquellos que trabajen en un servicio de atención al cliente. Crea un vínculo con el cliente en el que vea que eres capaz de entender su problema y que harás lo posible para solucionarlo.

- Humildad. Aparca tu prepotencia y toma las quejas de tus clientes como "críticas constructivas".

- Sé ágil y resolutivo. El tiempo apremia y más para tu cliente. iiNo te duermas en los laureles!! 
- Privacidad. Sé cuidadoso a la hora de pedir datos personales a tus clientes. Siempre privadamente.

- Agradecer. Finalmente, debéis mostrar vuestro agradecimiento por su fidelidad y demostrarle la importancia que el cliente tiene para vuestra empresa.

Y siempre se debe recordar que, la mejor tarjeta de visita que tu empresa puede tener, es un cliente satisfecho. (pp. 71-72).

\section{MÉTODO}

$\mathrm{L}$ a investigación se desarrolló con una metodología de tipo descriptivo, ya que mediante la misma buscó analizar y describir los datos y hechos más relevantes obtenidos tanto en la empresa línea Sindical Trans. Emperador, de manera que detalla la situación actual del nivel de la fidelización de los clientes para así poder dar soluciones asertivas.

El enfoque de la investigación fue mixto, cuantitativo, porque ayudo en la recolección, clasificación análisis e interpretación de datos como las planillas de pasajeros de la empresa de transporte línea Sindical Trans. Emperador, de la ciudad de Potosí y a la vez, se pudo establecer patrones de comportamiento mediante las encuestas aplicadas, en base en la medición numérica y el análisis estadístico. Y cualitativo, porque, la variable dependiente de la investigación, es decir, la fidelización del cliente, es más sensible a la cualificación, ya que, el mismo está relacionado con el estudio del comportamiento, gustos preferencias y la mente del cliente.

A su vez, el diseño de la investigación fue de tipo documental de campo, la primera, porque se requirió información sobre la fidelización de clientes y temas relacionados al marketing digital y lo segundo, porque se procedió al levantamiento de información primaria por medio de los instrumentos de recolección de datos.

Los métodos usados durante el desarrollo de la investigación fueron; análisis-síntesis, inductivo-deductivo, histórico-lógico y comparativo.

- Análisis-síntesis, los dos métodos se aplicaron con el fin de efectuar un análisis a profundidad de la recopilación bibliográfica como de los datos obtenidos de la empresa de transporte línea Sindical Trans. Emperador, de la ciudad de Potosí.

- Inductivo - deductivo, ya que estos dos métodos se complementaron mutuamente durante el proceso de investigación, el método inductivo fue el razonamiento que partiendo de casos particulares se eleva a conocimientos generales, mientras el método deductivo, conduce de lo general a lo particular.

- Histórico - lógico, fue de gran ayuda por lo que se halló un complemento y vinculación mutua, valiendo como guía en el contexto macro, meso y micro del objeto de estudio en el marco teórico que viene siendo fidelizar clientes.

- Comparativo, permitió hallar similitudes y diferencias entre otros operadores de servicio de transporte interdepartamental e interprovincial de la ciudad de Potosí. 
Por otro lado, las técnicas e instrumentos fueron, la entrevista y la encuesta.

- Entrevista, se obtuvo información sobre el problema planteado que es la fidelización de los clientes, partiendo de una guía semi estructurada la que se realizó a los socios de la línea Sindical Trans. Emperador de la ciudad de Potosí.

- Encuesta, se realizó a los clientes actuales y potenciales que adquieren el servicio de transporte que ofrece la línea Sindical Trans. Emperador de la ciudad de Potosí, haciendo uso de un cuestionario previamente.

La población de la investigación estuvo conforma por personas mayores de 18 años de la ciudad de Potosí, ya que este segmento de población es quien tiene poder de adquisición. Según el censo del 2012 asciende a un total de 115025 habitantes, por lo que fue la población en estudio.

En cuanto a la muestra, se utilizó la ecuación de poblaciones finitas, luego de los cálculos matemáticos se obtuvo el dato de 383 personas a ser encuestas.

\section{RESULTADOS}

\section{Datos obtenidos mediante el cuestionario}

El cuestionario fue aplicado 383 personas mayores de 18 años del municipio Potosí. Se obtuvo como resultado:

De acuerdo a la encuesta que se realizó en cuanto al género de los encuestados, en el Gráfico 1 , se revela que, el $56 \%$ equivalente a 214 personas son de sexo masculino y el restante $44 \%$ que equivale a 169 personas son de sexo femenino, lo que da un parámetro de equilibrio entre géneros sobre la aplicación del cuestionario.

El mayor porcentaje que fue encuestado con respecto al género fue del sexo masculino.

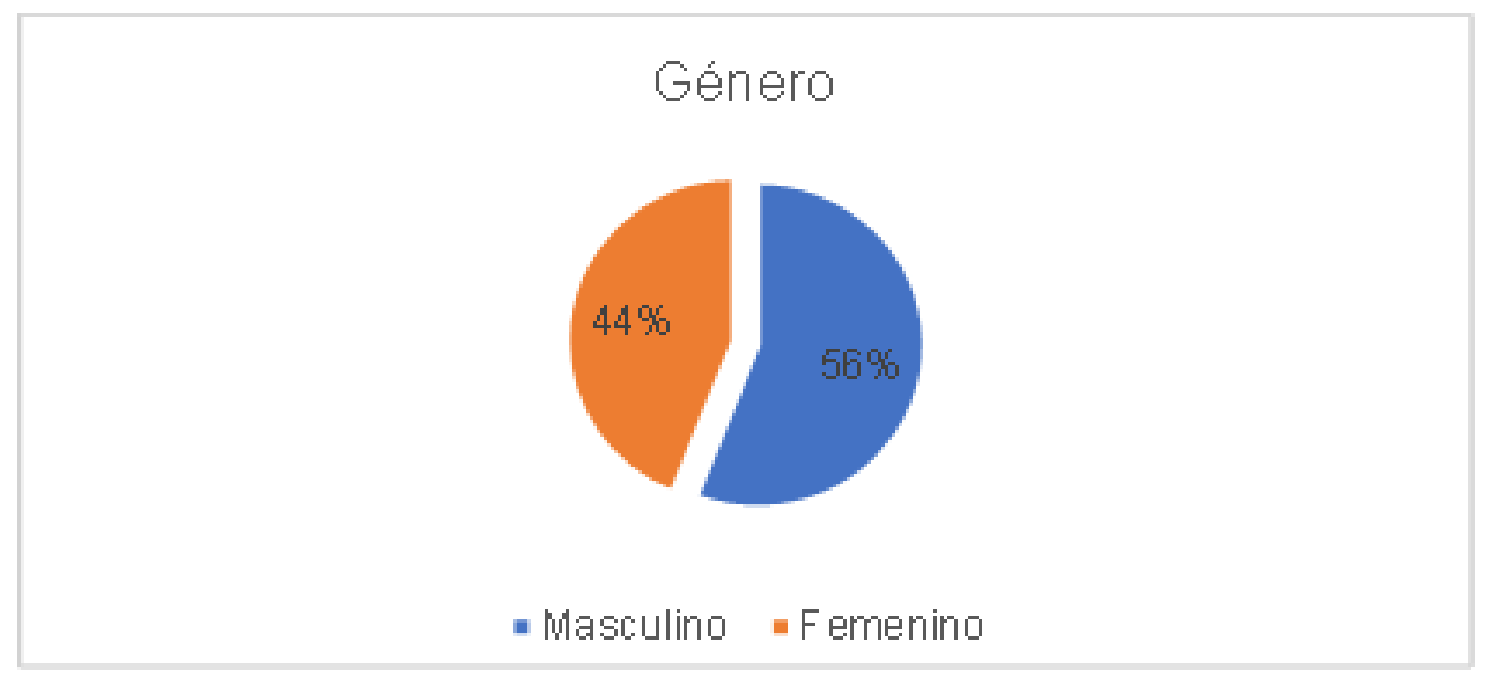

Gráfico 1. Género. 
En cuanto a lo que son las edades la mayoría de los encuestados, se muestra en el Gráfico 2 que, están entre los 18 hasta los 23 años con un $36 \%$ equivalente a 137 encuestados, entre los 24 hasta los 29 años en un 25\% que equivale a 96 encuestados, entre los 30 hasta los 35 años un $22 \%$ equivalente a 84 encuestados. Los resultados evidenciaron que todas las personas encuestadas están dentro el parámetro de población establecida por la muestra aleatoria simple.

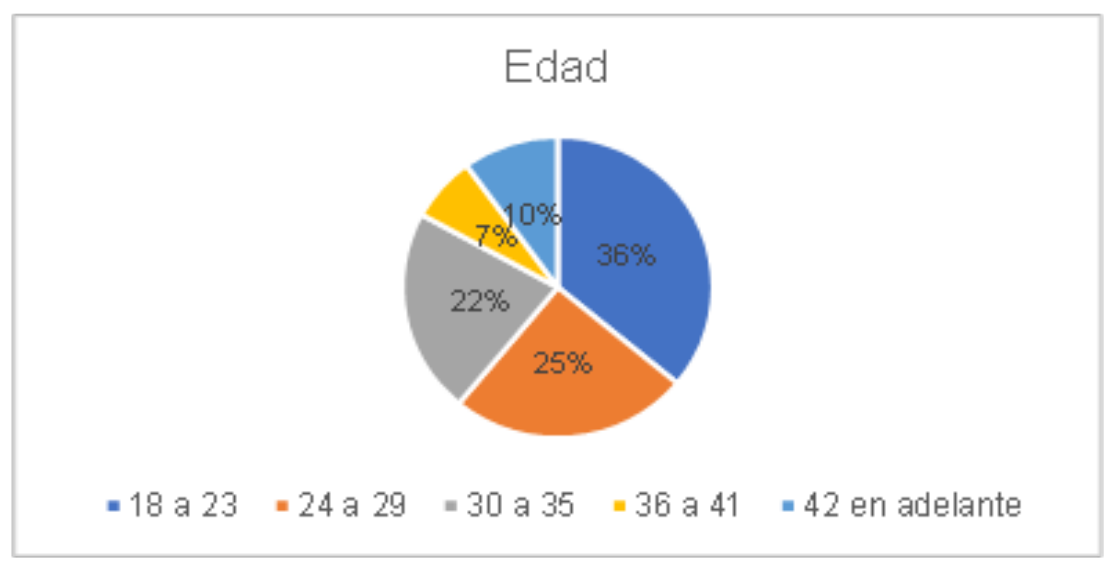

Gráfico 2. Edad.

Como se puede observar en el Gráfico 3, la empresa con mayor preferencia por los clientes que utilizan el servicio de transporte de la ciudad de Potosí hacia la ciudad de Chuquisaca es el servicio rápido con un 37\%, seguido por la empresa de transporte Trans Tin Dil Rey con un $23 \%$ y en tercer lugar se encuentra la línea Sindical Trans Emperador con un $20 \%$ equivalente a 47 encuestados.

Se evidencio la amplia flexibilidad de horarios, es la razón que la mayoría de los clientes encuestados prefieren como servicio de transporte a las empresas más conocidas como "los rapiditos".

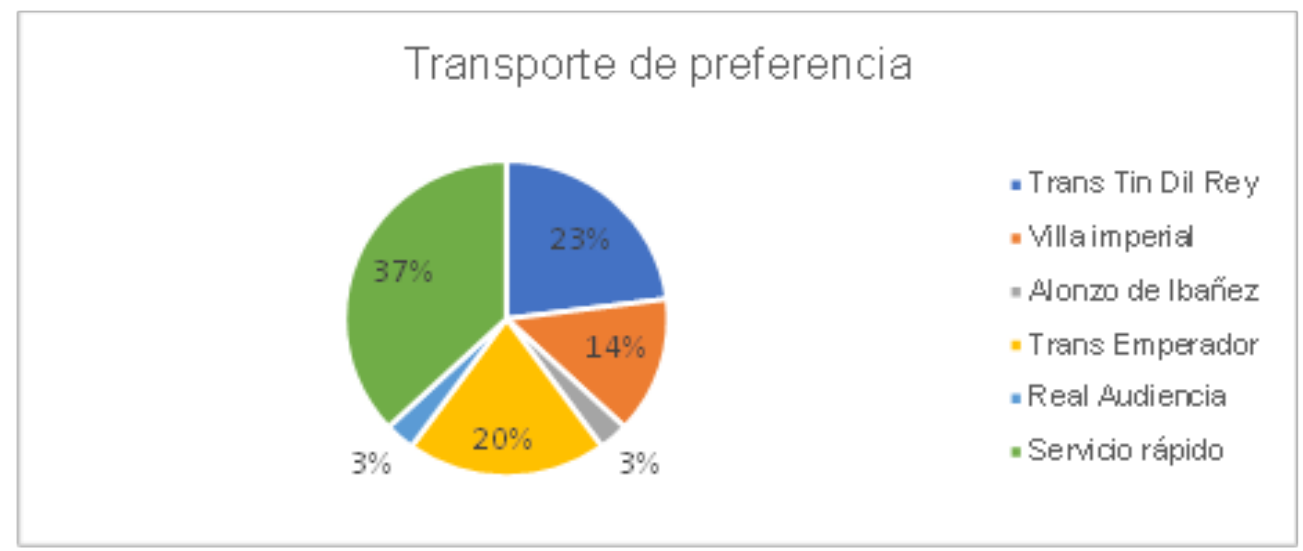

Gráfico 3. Empresas de transporte de su preferencia (Potosí- Sucre). 
Se aprecia en el Gráfico 4 que, de la población consultada respecto a que si utiliza o no los servicios de la línea Sindical Trans. Emperador el 58\% que equivale a 221 encuestados respondieron que no y el restante $42 \%$ equivalente a 162 encuestados respondieron que si hacen uso de los servicios que presta la línea Sindical Trans. Emperador.

De esta manera, se evidencio que, solo 162 personas son clientes reales y el resto de los encuestados llegarías a ser clientes potenciales.

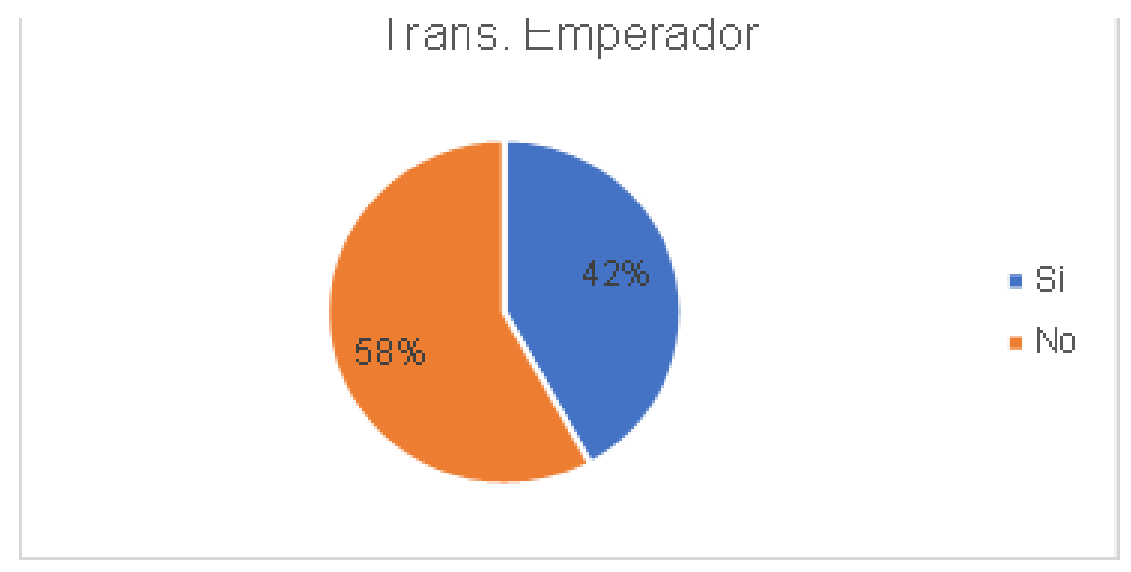

Gráfico 4. Uso del servicio transporte Emperador.

En el Gráfico 5, se muestra que, el $44 \%$ de los encuestados utiliza el servicio de transporte de pasajeros, y el $43 \%$ utilizan ambos servicios tanto el de transporte como el de encomiendas de todas las rutas que brinda la línea Sindical Trans. Emperador, y el 13 \%, hace uso exclusivamente del servicio de envío de encomiendas.

De acuerdo a este resultado se evidenció que, los clientes demandan en mayor porcentaje lo que viene siendo el servicio de transporte.

\section{Servicios con mayor frecuencia}

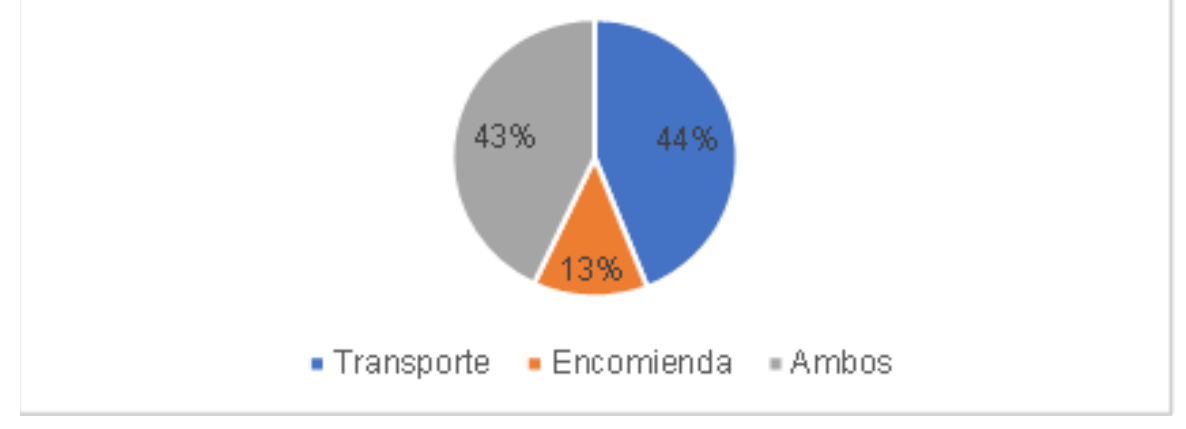

Gráfico 5. Servicios con mayor frecuencia. 
En el Gráfico 6, se muestra que, el $41 \%$ de las personas encuestadas indicaron que la calidad del servicio es regular, el $38 \%$ están conformes respecto a la calidad del servicio prestado y solo un $21 \%$ no se encuentra satisfecho con la calidad del servicio prestado que equivale a 33 clientes de las 162 personas que fueron encuestadas.

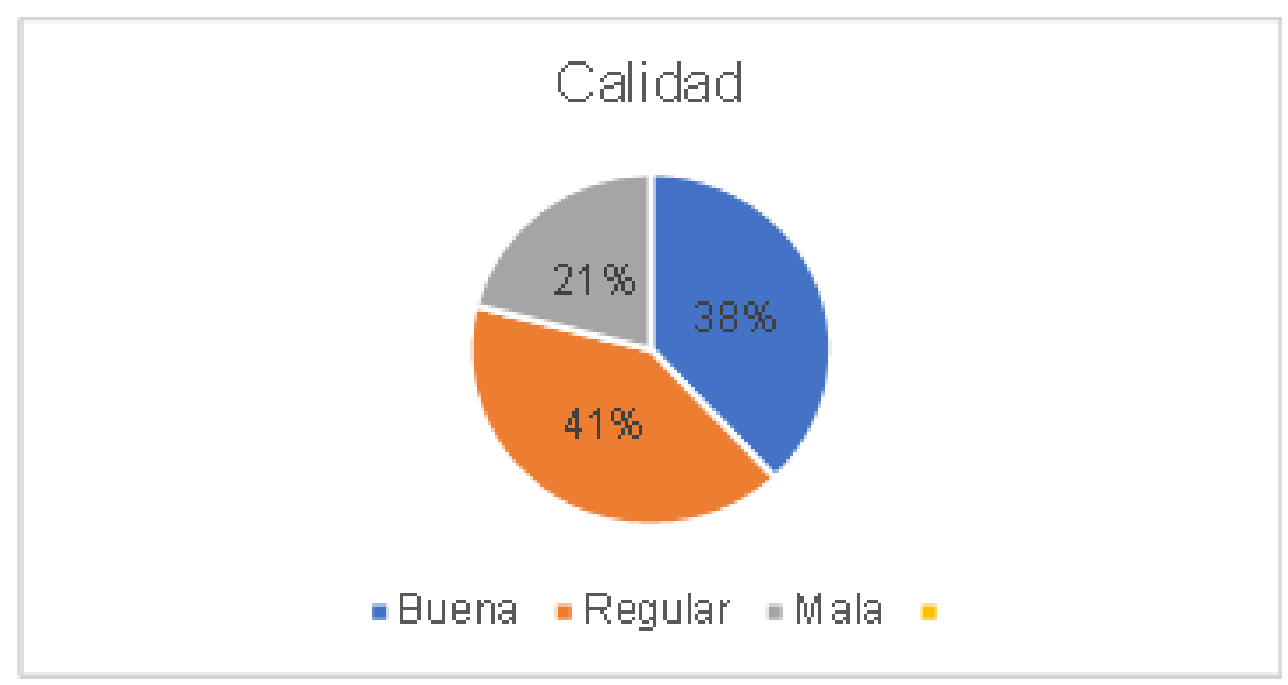

Gráfico 6. Calidad de servicio.

En el Gráfico 7, se muestra que, el mayor porcentaje de los clientes se siente que sus viajes son cómodos que es de $64 \%$, para el $36 \%$ que es la minoría indica que no se siente cómodo a la hora de viajar.

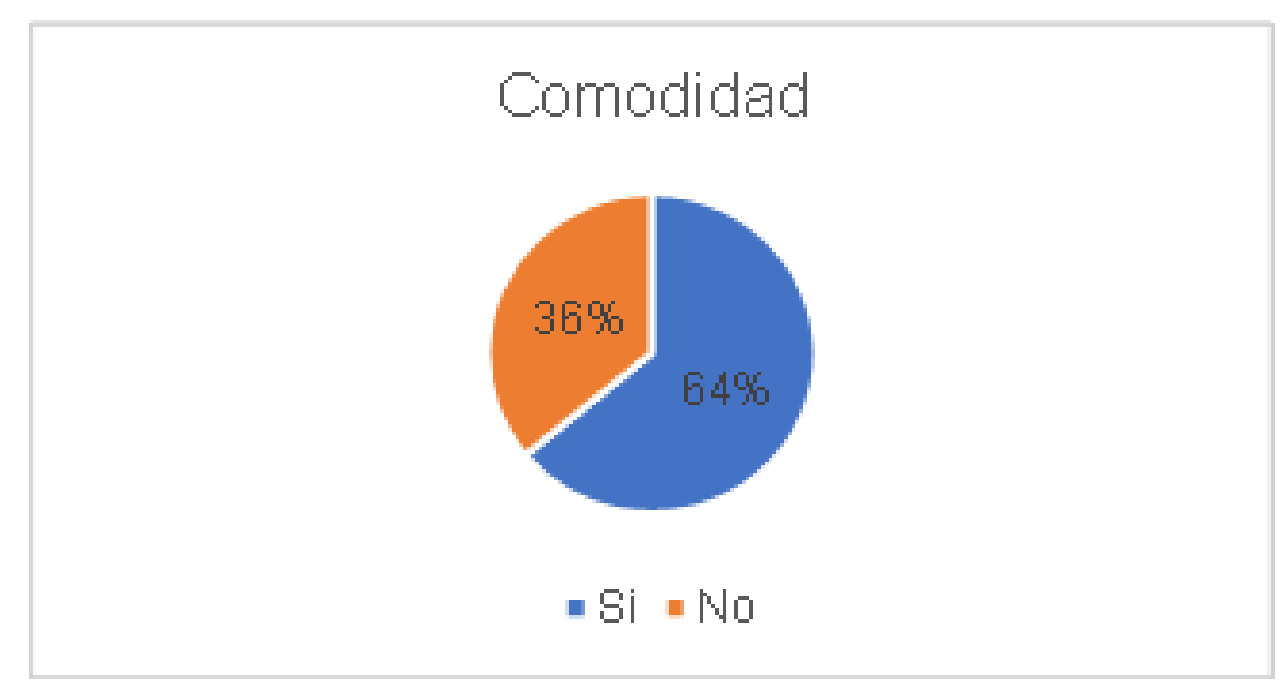

Gráfico 7. Comodidad. 
El Gráfico 8 muestra que, el $52 \%$ de los clientes sienten seguridad cuando hacen uso del servicio de transporte que brinda la línea Sindical Trans. Emperador y el $48 \%$ indica que no cuenta con los implementos necesarios para realizar los viajes.

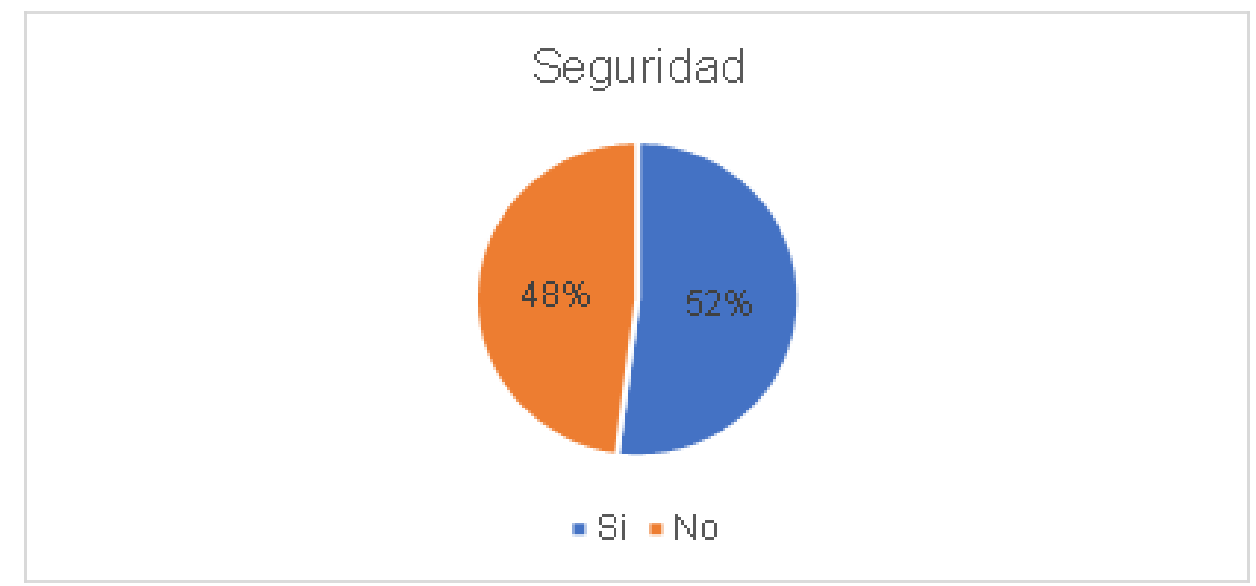

Gráfico 8. Seguridad

En el Gráfico 9, se puede apreciar que, el 58\% de los clientes encuestados de la línea Sindical Trans Emperador, no recomendaría el servicio que brinda para la población en general mientras que el $42 \%$ de las personas encuestadas, indican que si lo haría siendo una minoría.

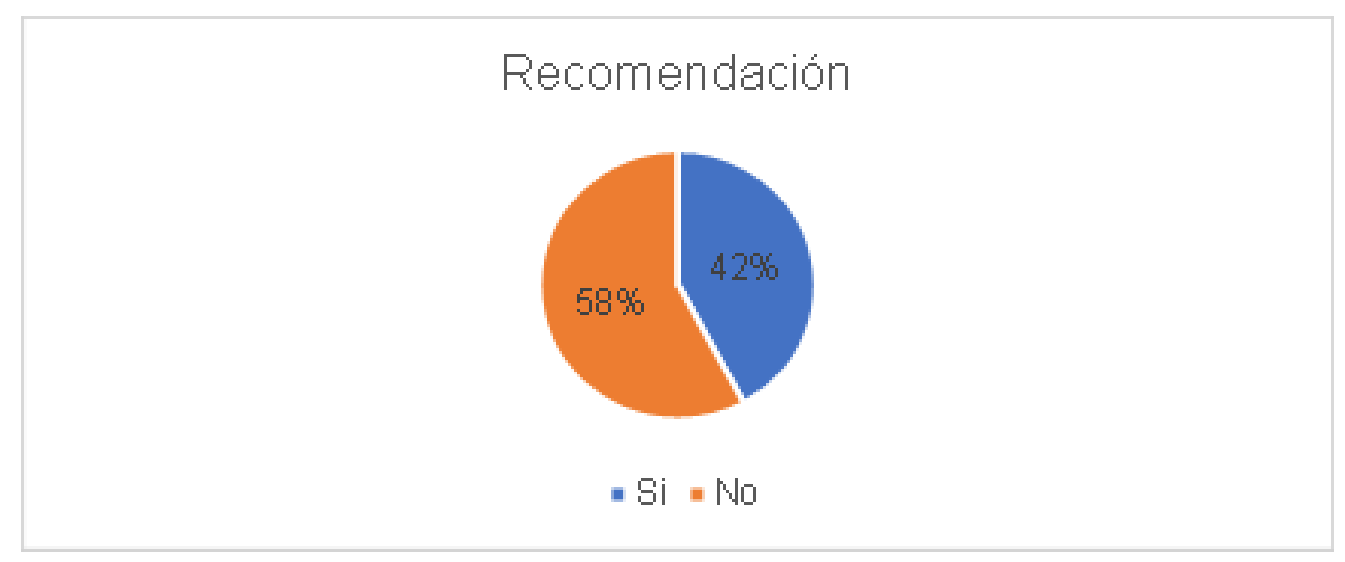

Gráfico 9. Recomendación. 
En este sentido, se evidencio que, los clientes no se sienten parte de la empresa por ende es mucho más difícil llegar a fidelizarlos. Porque al no satisfacer las necesidades y deseos que los clientes buscan en un servicio de transporte, los mismos buscaran en otra operadora los servicios que presta Trans. Emperador.

Por ende, se propone diversas tácticas de marketing digital para la fidelización de los clientes de la línea de transporte Emperador.

Propuesta de táctica marketing digital para la fidelización de clientes de la línea Sindical Trans. Emperador

En la investigación se planteó las siguientes tácticas de marketing digital para lograr la fidelización de clientes.

Página Web, el objetivo principal es dar confianza a los clientes. Esta Página Web no solo serviría para brindar información, sino que también se podría hacer reserva de pasajes como también la venta de pasajes mediante una transferencia electrónica, facilitando al cliente una forma de pago mucho más cómoda, por que el cliente ganaría tiempo. Ya que, si un cliente se sienta satisfecho contento con los servicios que se le preste será mucho más fácil fidelizar al cliente.

Estas son algunas razones por las cuales debería tener una página Web:

- Aumenta el reconocimiento y posicionamiento de una marca

- Hace incrementar las ventas

- Atrae más clientes

- Ayuda a fidelizar clientes actuales

Facebook para negocio, los objetivos más importantes son: interaccionar con los usuarios, fidelizar a sus clientes valorizar la marca y aumento de seguidores. Si la línea Sindical de Transporte Emprendedor crea una Página en Facebook (y la usa bien) tiene una ventaja sobre otra que, no la tenga. Por medio de Facebook, una página es por defecto pública, a diferencia de los perfiles de clientes personales, en los que sí se puede configurar la privacidad. Además, los clientes de Facebook solo tienen dos formas de interactuar con dicha página:

- "Me gusta": Al cliente les gusta pero puede que no siga tu página.

- "Seguir": Al cliente le gustas y te sigue; esto es, le llegan tus actualizaciones.

De esta forma la empresa tendrá más oportunidades de poder fidelizar a sus clientes. También servirá para poder transmitir anuncios que la línea Sindical Trans. Emperador mediante la página de Facebook.

Instagram para negocio, tiene como objetivo el compartir fotografías y videos para dar a conocer la empresa e interactuar con los clientes y los potenciales clientes. Si la línea Sindical Trans Emperador llegaría a estar presente en esta red social, tendría una ventaja competitiva frente a la competencia, ya que, se acercaría con más facilidad a los clientes y clientes potenciales, como también podrá compartir fotografías y videos relacionados con la empresa, de esta manera, poder facilitar el objetivo principal de la línea Sindical Trans Emperador que es fidelizar a sus clientes. 
Elaboración de un sistema de venta de pasajes, el objetivo principal de manejar un software para la venta de pasajes es que brinda una mejor base de datos y un mejor control respecto a la frecuencia de viajes de los clientes. Este software permitirá registrar las ventas, registro de cliente, conductores, rutas, horario, encomiendas y además, imprimirá un reporte semanal de los viajes, entregar un informe de cuanto se vendió, en que ruta, cual fue el autobús, quien lo conducía entre otras cosas más. No solo será de mucho beneficio para los socios de la línea Sindical Tran. Emperador, ya que, de igual manera permitirá un trabajo más simple para los colaboradores de la empresa, al contar con este software los encargados de las ventas y registro de pasajeros podrán generar reportes de todo tipo.

\section{CONCLUSIONES}

E n base al objetivo de la investigación se puede concluir que efectivamente se ha podido conceptualizar los principios y conceptos de la fidelización de clientes y marketing digital de la línea Sindical Trans. Emperador de la ciudad de Potosí, el cual permitió fundamentar teóricamente esta investigación, por lo que se logró establecer un posicionamiento teórico que permitió orientar hacia la propuesta,

Mediante la propuesta presentada se pretende contribuir a la fidelización de clientes de la línea Sindical Trans. Emperador de la ciudad de Potosí, para ello se debe implementar las tácticas de marketing digital, el cual le permitirá ofrecer un servicio de calidad a los clientes para que los mismos se sientan conformes y satisfechos con el servicio brindado por la empresa.

\section{REFERENCIAS}

Agüero, L. (2014). Estrategia de fidelización de clientes. Recuperado de https:// repositorio.unican.es/xmlui/bitstream/ handle/10902/4474/\%5B2\%5D\%20A g\%C3\%BCero\%20Cobo\%20L.pdf

Aguirre, M. (2006). Marketing en sectores específicos. Madrid, España: Ediciones Pirámides

Alcaide, J. (2002). Alta fidelidad: técnicas e ideas operativas para lograr la lealtad del cliente a través del servicio. Madrid, España: Esic Editorial

Bastos, A. (2006). Fidelización del cliente. Introducción a la venta personal y a la dirección de ventas. España: Ideas propias Editorial

Buchelli, E., y Cabrera, L. (2017). Aplicación de marketing digital y su influencia en el proceso de decisión de compra de los clientes. Recuperado dehttp://repositorio. upao.edu.pe/bitstream/upaorep/2998/1/RE_ ADMI_ESTRELLA.B UCHELLI_LUIS.CABRERA_ MARKETING.DIGITAL_DATOS.PDF
Censo (2012). Censo de población y vivienda 2012, Potosí, Bolivia

Escobar,S.(2013).Elmarketingdigitalylacaptación de clientes de la cooperativa de ahorro y crédito Universidad Técnica de Ambato Ltda. Recuperado de http://repositorio.uta.edu.ec/ bitstream/123456789/5887/1/62\%20MKT.pdf

Kotler, P., y Armstrong, G. (2003). Fundamentos de Marketing. ( $6^{a}$ ed.). México: Pearson Educación

Kotler, P., y Armstrong, G. (2012). Marketing (14a. ed). México: Pearson Education.

Lamb, Ch.; Hair, J., y McDaniel C. (2017). Marketing. (11a ed.). México: Cengage Learning

Merodio, J. (2000). Marketing en redes sociales. Recuperado de http://www. apatgn.org/c/document_library/get_ file?uuid=1fbd3475-a083-4e55- a28b657cf2030576\&groupld=10198 